\title{
Accuracy and Stability of Crossing Motion in Older Adults: With Focus on the Knee Joint
}

\section{Yusuke Maeda ${ }^{1 *}$ and Akihiko Hanafusa ${ }^{2}$}

${ }^{1}$ School of Health Sciences at Odawara, Department of Physical Therapy, International University of Health and Welfare, Japan

${ }^{2}$ Department of Bio-science and Engineering, College of Systems Engineering and Science, Shibaura Institute of Technology, Japan

\begin{abstract}
Background: Accidental falls are caused by sudden perturbations, and thus, quick and well-organized stepping responses are needed. Stepping is one of the most important motions for avoiding falls in elderly patients. We sought to analyze foot motion during the volitional steps of older adults and compare it with that of younger adults.
\end{abstract}

Methods: Eighteen healthy older adults (age: $70.8 \pm 3.2$ years) and 22 younger adults (age: $20.2 \pm 0.3$ years) were included in this study. The subjects crossed over a lateral obstacle 10 times, keeping the motion as consistent as possible. An infrared reflective marker, placed on the subjects' heels, recorded motion through a three-dimensional motion analysis system. A clinical sensory test for knee proprioception was performed and the difference between the target and the actual angle of the subjects was calculated using an electrical goniometer.

Results: The results of the motion analysis indicated that the trajectories of the heels in older adults were not consistent compared with that of young adults $(\mathrm{p}<0.05)$. Unfortunately, the clinical sensory test and the time-series analysis of knee angle change were unable to detect a decline in the proprioceptive function of the knee.

Conclusion: We believe that knee flexor muscles lack coordination in terms of eccentric contractions. This decline of coordination could cause the descending period to extend, which is the time from the top of the trajectory of the heel to the finish of the normalized motion. We also believe that the change in motion may indicate compensatory mechanisms for aging. This information can be useful for designing fall prevention exercises for the elderly population.

\section{Introduction}

The incidence of fall-related accidents is rising annually due to the increase in the aging population. Falls cause significant mental and physical problems that require a lengthy recovery period. Preventing falls can lead to the improvement of the quality-of-life (QOL) of older adults and reduce medical costs.

Previous studies have reported that lateral balance is important for preventing falls [1-3]. It is also related to falls in older adults [4]. Robinovitch reported that a lateral impact on the greater trochanter can lead to hip fractures in elderly patients [5]. The center of gravity (COG) is needed to maintain a stable standing posture and for returning to the central position of the limit of stability (LOS) when the COG is about to move out of the LOS. If the patient properly shifts his or her weight or finds a new base of support by taking a step, COG is maintained inside the LOS. However, the most frequent reason for falls during daily activities is incorrect weight shifting[6]. Therefore, a self-generated perturbation, such as a volitional side step in the lateral direction, should be investigated to determine whether it can prevent falls.

Proper crossing motion is needed to avoid stumbling on obstacles or stairs. However, it is difficult for elderly people to do crossing motions owing to their deteriorating sensory ability, spatial perception, and coordination. If they cannot raise their leg or foot as intended, they can trip and fall over. Important factors for achieving intended motion are motor and sensory functions. The constant feedback from the sensory system and a strong musculoskeletal system contributes to this motion. Crossing motion (volitional step) requires not only peripheral function (i.e. sensory and musculoskeletal system), but also a complex central neural system including the anticipatory postural control [7]. Sparto et al. reported that postural responses in older adults are variable, compared with young adults[ 8]. Caetano et al. reported that older adults were less accurate when performing stepping motions [9]. Therefore, it is necessary to clarify the characteristics of variable and inaccurate motion in older adults from a kinematical stand point. Consistent foot motion is needed to achieve clearance from an obstacle during the crossing motion.

The purpose of this study was to analyze foot motion during the volitional step of older adults. Moreover, we investigated the relationship between step motion and sensory function.

\section{Methods}

\section{Subjects}

The subjects were 18 healthy older adults ( 9 men and 9 women; age: $70.8 \pm 3.2$ years; height: $160.1 \pm 7.3 \mathrm{~cm}$; weight: $56.5 \pm 10.2 \mathrm{~kg}$ ) and 22 young adults ( 15 men and 7 women; age: $20.2 \pm 0.3$ years; height: $166.5 \pm 5.4 \mathrm{~cm}$; weight $58.4 \pm 9.1 \mathrm{~kg}$ ). The subjects had no

"Corresponding Author: Dr. Yusuke Maeda, School of Health Sciences at Odawara, Department of Physical Therapy, International University of Health and Welfare, 1-2-25 Shiroyama, Odawara City, Kanagawa, 250-8588, Japan, Tel: +81(465) 21-6671, Fax: +81(465) 21-6745; E-mail: y.maeda@iuhw.ac.jp

Citation: Maeda Y, Hanafusa A (2018) Accuracy and Stability of Crossing Motion in Older Aadults: With Focus on the Knee Joint. Int J Phys Ther Rehab 3: 143. doi: https://doi.org/10.15344/2455-7498/2018/142

Copyright: (c) 2018 Maeda et al. This is an open-access article distributed under the terms of the Creative Commons Attribution License, which permits unrestricted use, distribution, and reproduction in any medium, provided the original author and source are credited. 
neurological disorders or orthopedic history with respect to their lower limbs. Subjects were provided a carefully written explanation of the experimental purpose, method, and privacy protection in advance of the experiment. They submitted their written informed consent to participate in this study. This study was approved by the Ethical Committee of Mejiro University.

\section{Procedure}

The subjects were asked to cross over a lateral obstacle with unrestricted motion velocity and landing position. They crossed their arms in front of their chest and looked at a target on the wall. They were asked to try not to look at their feet. They tried to repeat this motion 10 times and were asked to keep each motion as consistent as possible with the last. They practiced several times and decided on the landing position of the foot. The height of the obstacle was $20 \%$ of the subjects' heights. The obstacle consisted of a wood and iron pipe.

We conducted a sensory test to examine the position sense of the right knee. Subjects lay on their stomach, while the examiner held their right lower leg and stabilized the knee joint up to target angle. Subjects flexed their left knee, similar to right knee flexion, while the examiner held up the right knee. The differences between the angles of the right and left knee were recorded. The target angles were $20^{\circ}$, $40^{\circ}, 60^{\circ}$, and $80^{\circ}$. The position sense test was conducted 3 times for each target angle.

\section{Experimental set up}

Crossing motion was recorded using a three-dimensional analysis system (DKH Co. Ltd., Japan). Reflective markers 14-mm in diameter were placed on the right side of the anterior superior iliac spine, the greater trochanter, knee joint center, the lateral malleolus, the heel, and the head of the fifth metatarsal. Two video cameras were used for motion analysis. The sampling rate of the cameras was $100 \mathrm{~Hz}$.

An electrical goniometer was used to examine the knee angle. The proximal part of the goniometer was attached to the lateral thigh along the axis (great trochanter knee center). Similarly, the distal part was attached to the lateral part of the lower leg (fibula). A measurement of zero degrees was defined as fully extended. All the equipment was synchronized and operated simultaneously.

\section{Data analysis}

Frame-Dias (DKH Co. Ltd., Japan) was used to analyze knee motion and heel trajectory. The angle changes of the knee and heel motion were calculated using a time-series analysis.

The average of trajectory curves of heel and angle change of knee motion were calculated by analyzing the degree of coincidence between the ten trials using the following methods by MATLAB (Math works, Inc. USA). First, the start and end points of the curve were defined as the moment that the heel was off the ground and made contact, respectively, and normalized from 0 to 1 . To calculate the average trajectory of the curves, the curves were approximated using cubic spline function, and the data were resampled at regular intervals. Figure 1 shows the method of calculation or the sum of error distance of the heels. The sum of the error angles of the knee angle were analyzed using the same method.

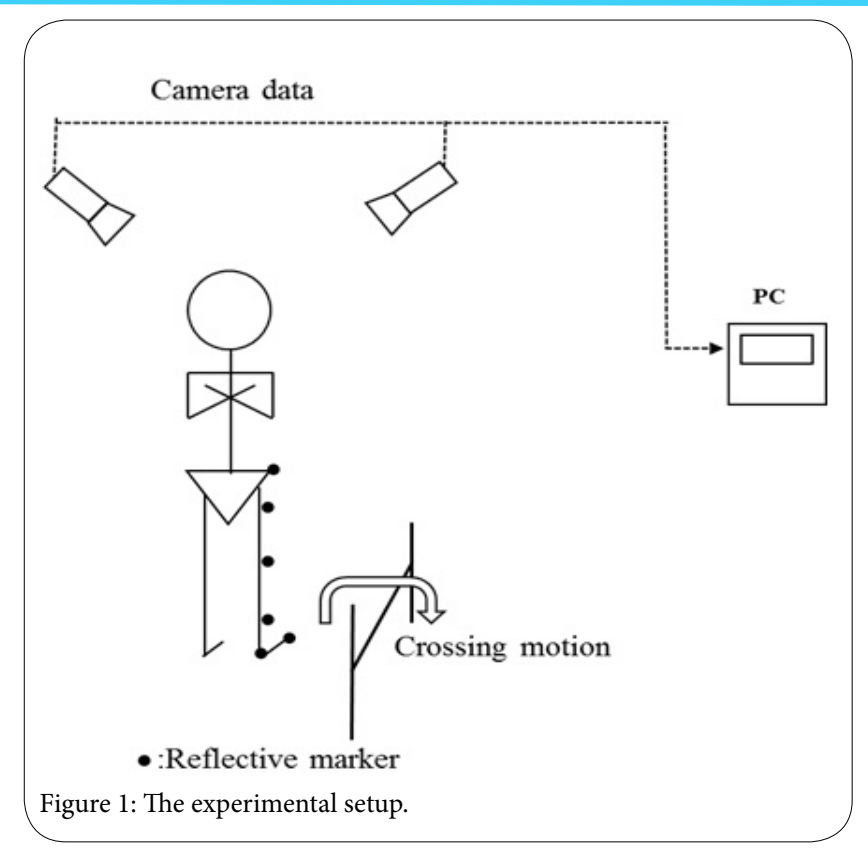

The peak time was defined as the time between the taking off of the motion leg to the top of the trajectory, and it indicated the percentage.

Errors from the target angle were recorded in each angle $\left(20^{\circ}, 40^{\circ}\right.$, $60^{\circ}$, and $80^{\circ}$ ) of the sensory test. The average of errors among the three trials was used.

\section{Statistical analysis}

The differences in each parameter of sum of error distance, peak time, and sensory test between two groups were compared using the t-test. A p value of $<0.05$ was considered statistically significant. All statistical analyses were conducted using SPSS 20.0 software (SPSS, Tokyo, Japan).

\section{Results}

Table 1 shows that the peak times of the motion of older adults were statistically shorter than that of young adults $(\mathrm{p}<0.05)$. Table 2 shows the sum of error distance in older adults and was statistically larger than that of young adults ( $\mathrm{p}<0.05)$. Table 3 shows that the error of angle had no statistical significance. Table 4 shows the error of sensory test of each target angle and had no statistically significant difference.

\begin{tabular}{|c|c|c|c|}
\hline & Older adults & Younger adults & \\
\hline Peak time & $44.6 \pm 3.7$ & $48.1 \pm 2.8$ & ${ }^{*} \mathrm{p}<0.05$ \\
\hline $\begin{array}{l}\text { Table 1: The } \\
\text { Data are pre } \\
\text { *: Significan }\end{array}$ & $\begin{array}{l}\text { ak time }(\%) \text {. } \\
\text { ted as mean } \pm \\
\text { oup difference }\end{array}$ & & \\
\hline
\end{tabular}

\begin{tabular}{|l|l|l|l|}
\hline & Older adults & Younger adults & \\
\hline Averaged error distance & $0.0292 \pm 0.015$ & $0.0218 \pm 0.005$ & ${ }^{*} \mathrm{p}<0.05$ \\
\hline Table 2: The averaged error distance $(\mathrm{m})$. \\
Data are presented as mean \pm SD. \\
${ }^{*}$ : Significant group distance.
\end{tabular}


Citation: Maeda Y, Hanafusa A (2018) Accuracy and Stability of Crossing Motion in Older Aadults: With Focus on the Knee Joint. Int J Phys Ther Rehab 3: 143. doi: https://doi.org/10.15344/2455-7498/2018/142

Page 3 of 5

\begin{tabular}{|l|l|l|l|}
\hline & Older adults & Younger adults & \\
\hline Averaged error angle & $10.8 \pm 3.7$ & $9.0 \pm 4.6$ & N. S. \\
\hline
\end{tabular}

Table 3: The averaged error angle (deg).

Date are presented as mean \pm SD.

N. S.: Not Significant.

Figure 2-6 show the trajectory of heel markers of a typical older and younger adult, respectively. Each figure shows all 10 trial trajectories.

\begin{tabular}{|l|l|l|l|}
\hline Target angle & Older adults & Younger adults & \\
\hline $20(\mathrm{deg})$ & $10.8 \pm 7.2$ & $7.3 \pm 4.6$ & N. S. \\
\hline $40(\mathrm{deg})$ & $12.0 \pm 9.7$ & $9.7 \pm 6.1$ & N. S. \\
\hline $60(\mathrm{deg})$ & $13.8 \pm 12.4$ & $9.9 \pm 6.4$ & N. S. \\
\hline $80(\mathrm{deg})$ & $14.3 \pm 13.7$ & $9.7 \pm 7.8$ & N. S. \\
\hline
\end{tabular}

Table 4: The error in the sensory test (deg).

Data are presented as mean \pm SD.

N. S.: Not Significant.

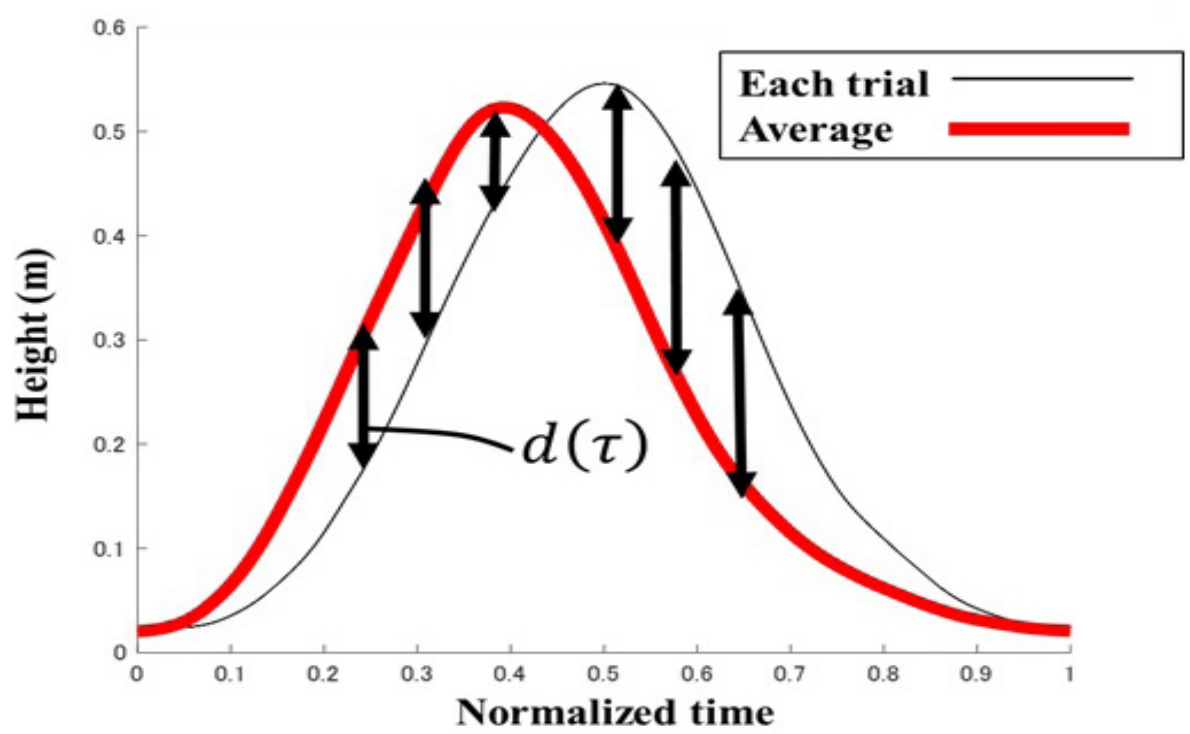

Figure 2. The method used to calculate the averaged error distance and angle is depicted. $\mathrm{d}(\tau)$ is as indicated below. $d(\tau)=|f(\tau)-\overline{f(\tau)}|$

$\mathrm{d}(\tau)$ represents the gap between each trial and the average at time $\tau$. In addition, $(\overline{)}$ is the average of $\mathrm{d}(\tau)$ across the ten trials, normalized from 0 to 1 .

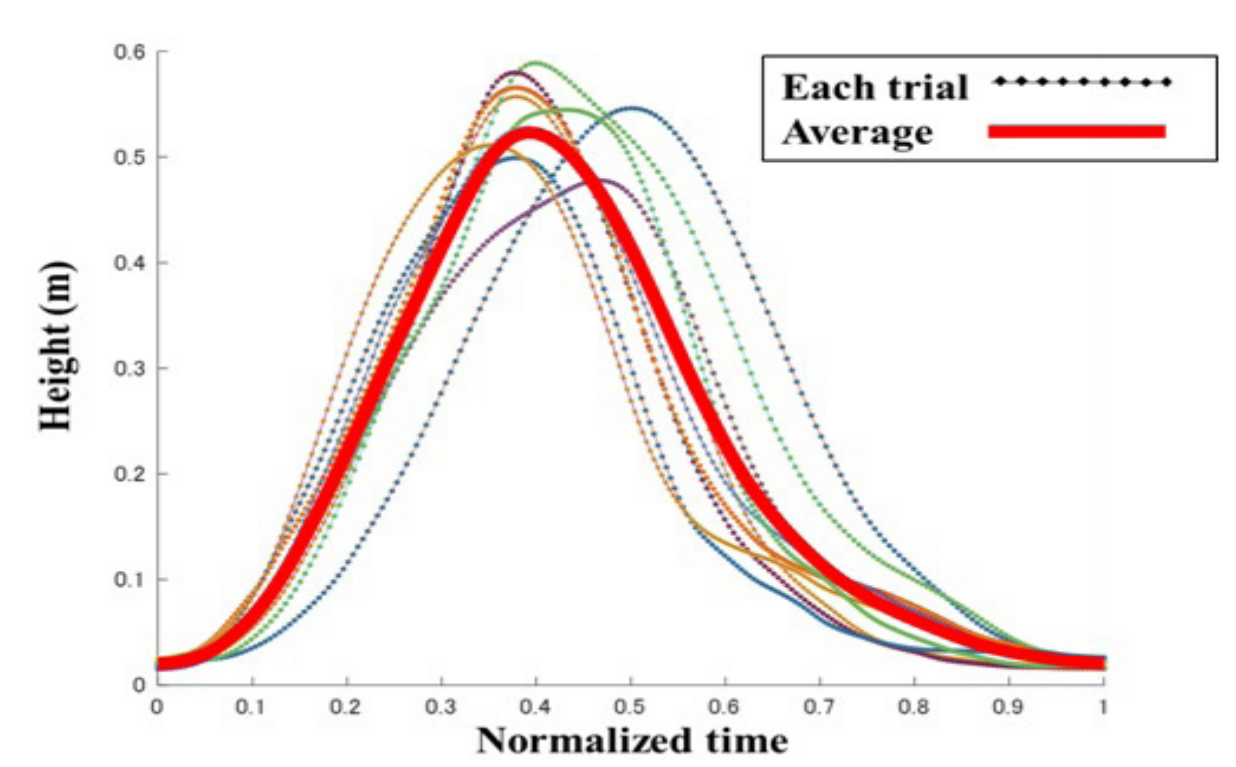

Figure 3: Typical trajectory of the heel marker in an older adult. 
Citation: Maeda Y, Hanafusa A (2018) Accuracy and Stability of Crossing Motion in Older Aadults: With Focus on the Knee Joint. Int J Phys Ther Rehab 3: 143. doi: https://doi.org/10.15344/2455-7498/2018/142

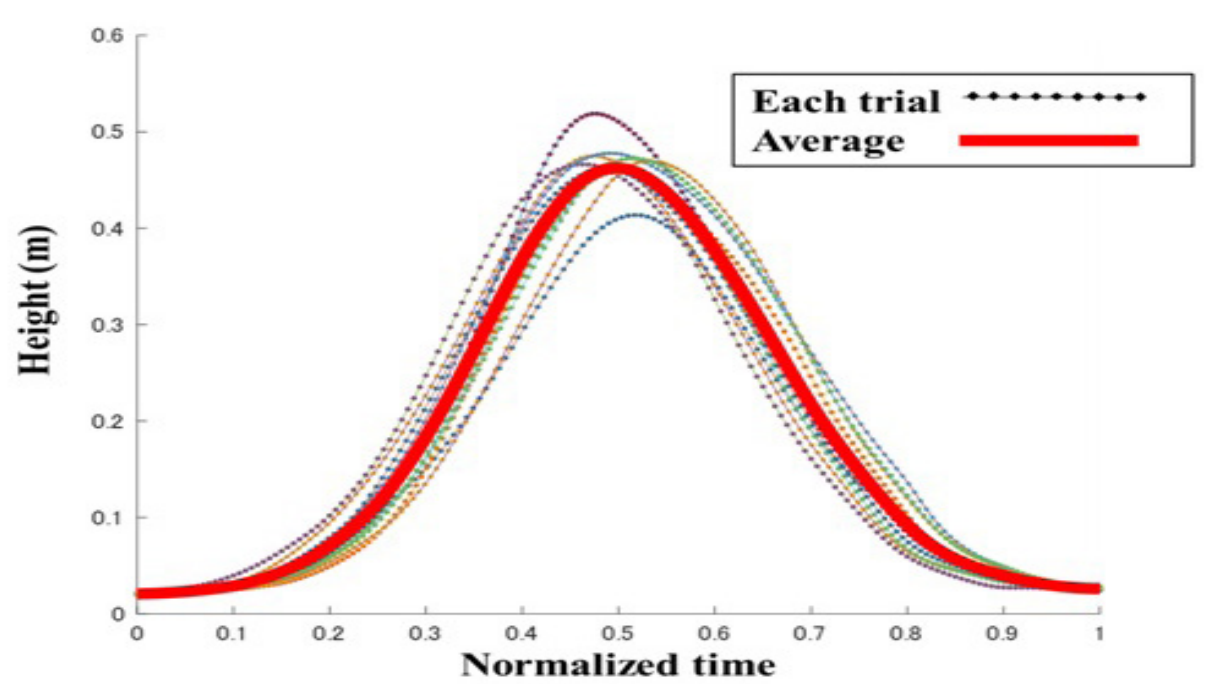

Figure 4: Typical trajectory of the heel marker in a younger adult.

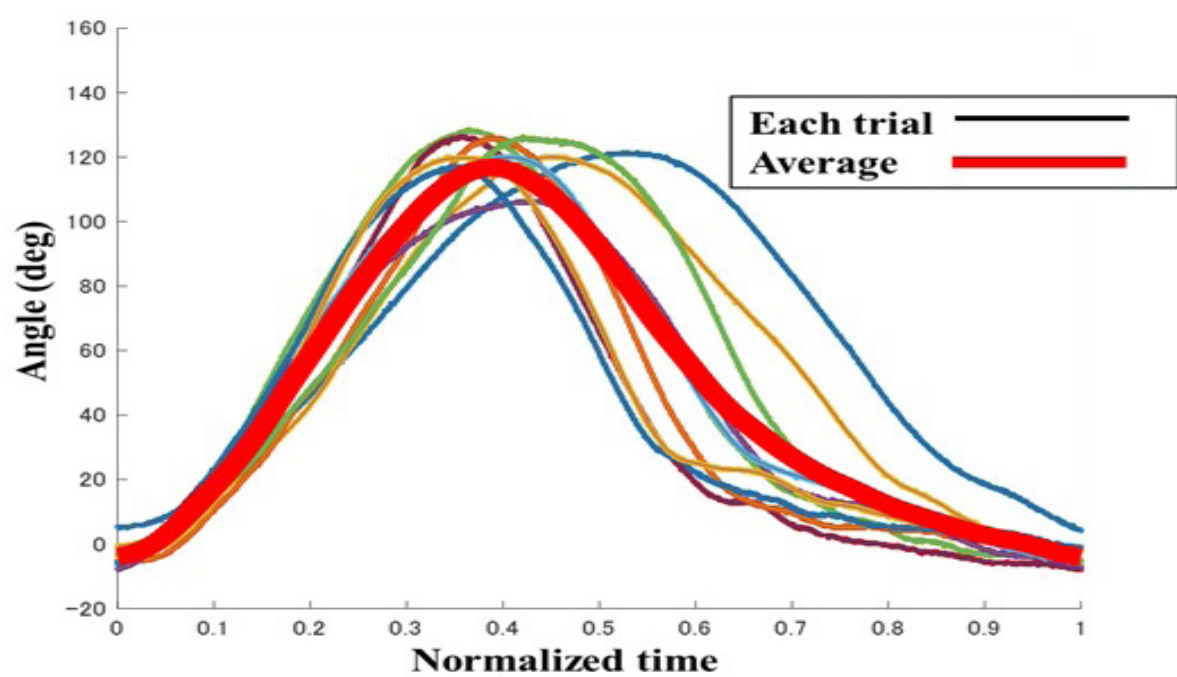

Figure 5: Typical knee angle change in an older adult.

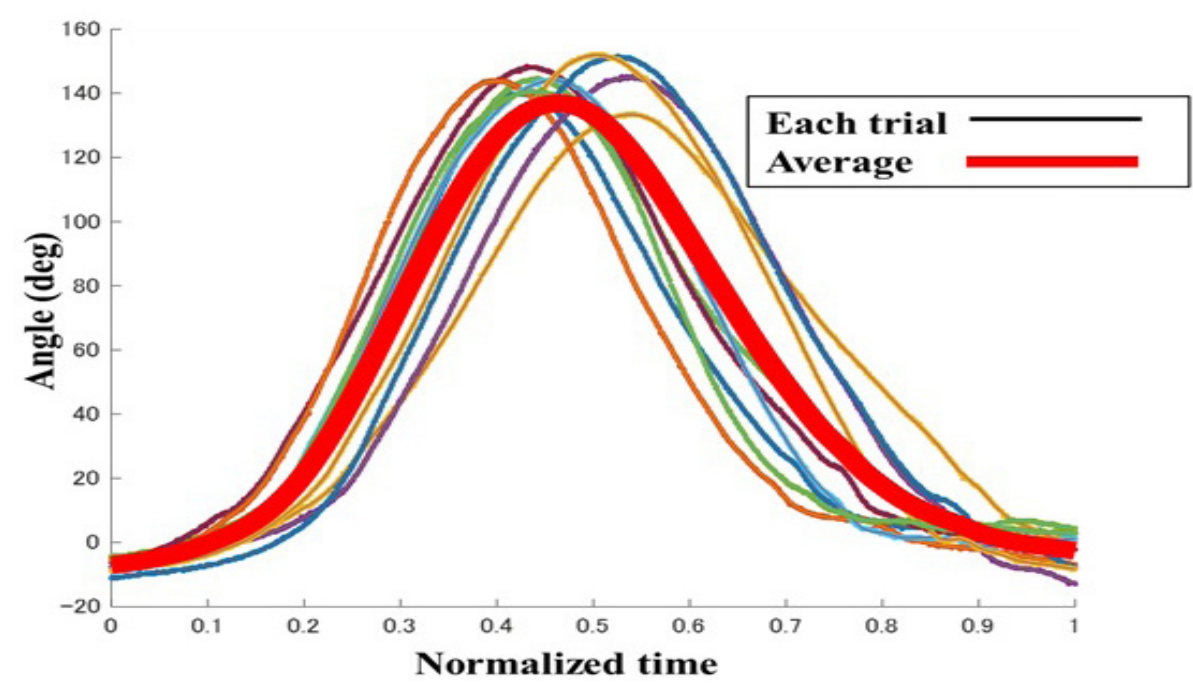

Figure 6: Typical knee angle change in a younger adult. 
Citation: Maeda Y, Hanafusa A (2018) Accuracy and Stability of Crossing Motion in Older Aadults: With Focus on the Knee Joint. Int J Phys Ther Rehab 3: 143. doi: https://doi.org/10.15344/2455-7498/2018/142

Page 5 of 5

\section{Discussion}

The sum of error distance results showed that the foot motions during the steps of older adults are inconsistent. We believe that as sum of error distance increases, the consistency of the motion decreases. Therefore, the crossing motion in older adults may vary widely and lack accuracy. In addition, we believe that motion may change due to aging.

Well-controlled concentric and eccentric contraction, especially of knee joint, should be used for this type of consistent motion. Sensory and motor function and the coordination of many muscles are needed to achieve this motion. One of the most important parts of the sensory system may be proprioception of the knee joint during consistent crossing motion. In a previous study, researchers found that proprioceptive signals encode joint angles [10]. However, these functions generally deteriorate in older adults. We did not observe a decline of proprioception of the knee joint and inconsistency of knee angle during motion, respectively, according to the results of the sensory test and sum of error angles. A slight decline of proprioception could not be detected through the clinical sensory examination of knee joint of older adults. In terms of motor function, eccentric motions are more complex and difficult to control compared to concentric contractions, especially for older adults [11]. However, the force of eccentric contraction is maintained in older adults [12]. Therefore, we believe that older adults are less able to adjust their intended contraction force. This decline may be related to the inconsistencies we observed in their motion and may cause falls. Therefore, exercises that improve accuracy and coordination are needed to prevent falls in older adults.

Furthermore, we found that the ascending period became short, while the descending period became extended in older adults. Therefore, they would pay more attention to landing than young adults in the descending period. A previous study showed that older adults often step before they need to, and therefore, it is likely that cognitive factors also influence the stepping response as seen in the extended descending phase of our study [13]. They may change their motion as they recognized their deteriorating ability for eccentric contraction. This may be an automatically compensated motion during aging. We believe that the change in motion is difficult in older adults and indicates a compensatory mechanism that is part of aging.

\section{Conclusion}

We collected the three-dimensional kinematic data of heel trajectory for crossing motion. Older adults performed inaccurate and inconsistent motion compared with young adults. Moreover, their actual physical motion differed from that of young adults. These results indicate that older adults cannot smoothly adjust their motion during eccentric contraction and cannot compensate for their decline in function by changing their motion. It is important to identify changes of motion in elderly patients in the clinic. Rehabilitation to improve coordination is needed in older adults to acquire accurate and stable motion.

\section{Competing Interests}

The authors declare that they have no competing interests.

\section{References}

1. Mansfield AMaki BE (2009) Are age-related impairments in changein-support balance reactions dependent on the method of balance perturbation? J Biomech 42: 1023-1031.

2. Lord SR, Rogers MW, Howland A, Fitzpatrick R (1999) Lateral Stability, Sensorimotor Function and Falls in Older People. J Am Geriatr Soc 47: 1077-1081.

3. Smeesters C, Hayes WC, Mcmahon TA (2001) Disturbance type and gait speed affect fall direction and impact location. J Biomech 34: 309-317.

4. Schrager MA, Kelly VE, Price R, Ferrucci L, Shumway-CookA, et al. (2008) The effects of age on medio-lateral stability during normal and narrow base walking. Gait Posture. 28: 466-471.

5. Robinovitch SN, Hayes WC, Mcmahon TA (1991) Prediction of Femoral Impact Forces in Falls on the Hip. J Biomech Eng 113: 366-374.

6. Robinovitch SN, Feldman F, Yang Y, Schonnop R, Leung PM, et al. (2013) Video capture of the circumstances of falls in elderly people residing in long-term care: an observational study. Lancet 381: 47-54.

7. Chu YH, Tang PF, Chen HY, Cheng CH (2009) Altered muscle activation characteristics associated with single volitional forward stepping in middleaged adults. Clinical Biomechanics 24: 735-743.

8. Sparto PJ, Jennings JR, Furman JM, Redfern MS (2014) Lateral step initiation behavior in older adults. Gait Posture 39: 799-803.

9. Caetano MJD, Lord SR, Schoene D, Pelicioni PHS, Sturnieks DL, et al (2016) Age-related changes in gait adaptability in response to unpredictable obstacles and stepping targets. Gait Posture 46: 35-41.

10. Beckmann YY, Çiftçi Y, Ertekin C (2013) The detection of sensitivity of proprioception by a new clinical test: The dual joint position test. Clin Neurol Neurosurg. 115: 1023-1027.

11. Yao WX, Li J, Jiang Z, Gao JH, Franklin CG, et al. (2014) Aging interferes central control mechanism for eccentric muscle contraction. Front Aging Neurosci. 6: 86.

12. Roig M, Macintyre DL, Eng JJ, Narici MV, Maganaris CN, et al. (2010) Preservation of eccentric strength in older adults: Evidence, mechanisms and implications for training and rehabilitation. Exp Gerontol. 45: 400-409.

13. Rogers MW, Hedman LD, Johnson ME, Martinez KM, Mille ML, et al. (2004) Triggering of protective stepping for the control of human balance: age and contextual dependence. Cognitive Brain Research. 16: 192-198. 